\title{
Are there long-term benefits of donepezil in Alzheimer's disease?
}

Courtney C, Farrell D, Gray R, Hills R, Lynch L, Sellwood E, et al; AD2000 Collaborative Group. Long-term donepezil treatment in 565 patients with Alzheimer's disease (AD2000): randomised double-blind trial. Lancet 2004;363:2105-15.

Background: Second-generation cholinesterase inhibitors (donepezil, galantamine and rivastigmine) have been shown to have beneficial effects on cognitive, global and daily functioning lasting 6-12 months in patients with mild to moderate Alzheimer's disease (AD) in double-blind, randomized controlled trials (RCTs), ${ }^{1,2}$ with continued benefits apparent in open-label extensions over 3-4 years. ${ }^{3}$ Doubleblind data concerning their long-term utility are lacking.

Design: The investigators set out to conduct a double-blind, longterm RCT of donepezil for the treatment of $\mathrm{AD}$ in a family practice setting, using pragmatic end points; the study was funded by a UK National Health System regional authority, free of pharmaceutical industry sponsorship. The original target of $3000 \mathrm{pa}-$ tients was revised to 566 owing to recruitment problems (the final sample was $565 ; 1$ patient withdrew consent before treatment). The 2 primary outcomes were entry into institutional care and progression of disability (defined as loss of 2 of 4 basic or 6 of 11 instrumental activities on the Bristol Activities of Daily Living Scale [BADLS]). Secondary measures included results of other objective psychometric tests (e.g., the Mini-Mental Status Exam [MMSE]), functional ability on the BADLS, behavioural symptom and caregiver burden scales, and costs of care.

$\stackrel{\sim}{a}$ Eligible patients met DSM-IV

criteria for $\mathrm{AD}$ with or without vascular dementia, and clinicians had to be "substantially uncertain" that patients would benefit from taking donepezil. After a 12-week compliance run-in, 486 patients were randomly reas- signed and followed up quarterly for 48 weeks. After a 6-week wash-out period, remaining patients continued for another 48 weeks. The 486 participants provided $90 \%$ power at $p<0.05$ to detect a 6-month annual delay to entry into institutional care and an effect size of 0.3 standard deviations on the secondary measures in the intervention arm versus the placebo arm.

Results: A total of 293 patients $(60 \%)$ completed the first year and $111(23 \%)$ the second year of follow-up. At 2 years, no significant differences were found between the 2 groups in the primary outcome measures. Of the secondary measures, the mean MMSE score at 2 years was 0.8 points higher in the donepezil group than in the placebo group (95\% confidence interval [CI] $0.5-1.2, p<0.0001)$, and the mean BADLS score for functionality was 1.0 point higher in the donepezil group than in the placebo group (95\% CI 0.5-1.6, $p=0.0004)$. No other measures or subgroup analyses showed significant differences. The mean annual net cost was $£ 498$ higher in the donepezil group. Serious adverse events and deaths were similar in both groups, but by 1 year, more of the patients in the donepezil group than in the placebo group discontinued treatment because of side effects ( $7 \%$ v. $3 \%$ ).

Commentary: The main flaw of this study was that it was underpowered. The power, calculated on the basis of 486 subjects being in the final randomization for the long-term study, was undermined by a $40 \%$ dropout rate by year 1 and $77 \%$ dropout rate by year 2. Although dropout num- bers were similar in both groups, the authors did not specify how many patients crossed over to the other treatment arm. This could be a major confounder. Furthermore, although demographic characteristics, baseline scores and comorbid diagnoses were balanced in the initial sample, no such data were given for the subjects who were eventually randomly assigned in the long-term study. Differences in baseline characteristics between the $2 \mathrm{fi}$ nal groups (e.g., pre-existing cerebrovascular disease and psychosis, which had not been excluded in order to increase sample generalizability) could have affected the outcomes.

Dosing and treatment interruptions did not accord with usual practice. The authors do not explain why subjects were washed out for 6 and 4 weeks after each 48-week treatment period. Previous trial data suggest this could attenuate response and patients might not recoup gains made on treatment. Also, the 5and 10-mg doses were analyzed together, presumably because of sample attrition. In clinical practice, the treatment goal is $10 \mathrm{mg}$ if tolerated, and $5 \mathrm{mg}$ is considered suboptimal; therefore, inclusion of patients taking the lower dose may have diluted the treatment effects.

Another concern is the use of doctors' "substantial uncertainty" about drug benefit as a premise for enrolment and how this was conveyed to patients and their physicians. Setting realistic expectations - a delay of 6-12 months in disease progression, possibly longer in some cases is important for appropriate prescription of cholinesterase inhibitors. Making doubt the basis for recruitment may have subtly biased patient selection, expectations, tolerance of side effects and dropout rates, and potentially influenced outcomes. The decision to enter into institutional care, al- 
though apparently a hard end point of the study, is a complex reflection of social, cultural, caregiver and patient factors.

Practice implications: Despite this study being well intentioned, there were problems with patient recruitment and retention. As such, the calculation for statistical power was performed posthoc; the treatment effects that the study was powered to detect based on patient enrolment were small to medium. Inadequately powered to detect more meaningful clinical differences, the study cannot be interpreted positively or negatively - absence of proof is not proof of absence. Hence, the study neither refutes nor strengthens existing guidelines, which recommend a trial of cholinesterase inhibitors in cases of mild to moderate AD. Clearly what is needed are better measures to set expectations and evaluate outcomes, data analysis independent - as in this study - of pharmaceutical sponsors and more effective, diseasemodifying interventions.

\section{Sandra E. Black}

John P. Szalai (deceased)

Department of Medicine

(Neurology)

Department of Research Design and Biostatistics

Sunnybrook and Women's College

Health Sciences Centre

University of Toronto

Toronto, Ont.
Competing interests: None declared for John Szalai. Sandra Black received honoraria for CME lectures and ad-hoc consultations from the manufacturers of donepezil (Pfizer Canada Inc.), rivastigmine (Novartis Pharmaceuticals Inc.) and galantamine (Janssen-Ortho Inc.).

\section{References}

1. Lanctôt KL, Herrmann N, Yau KK, Khan LR, Liu BA, LouLou MM, et al. Efficacy and safety of cholinesterase inhibitors in Alzheimer's disease: a meta-analysis. CMAf 2003;169(6): 557-64.

2. Mohs RC, Doody RS, Morris JC, Ieni JR, Rogers SL, Perdomo CA, et al. A 1-year, placebo-controlled preservation of function survival study of donepezil in AD patients. Neurology 2001;57(3):481-8.

3. Raskind MA, Peskind ER, Truyen L, Kershaw P, Damaraju CV. The cognitive benefits of galantamine are sustained for at least 36 months: a longterm extension trial. Arch Neurol 2004;61:252-6. 